Tom 25 № 4

УДК 512.542 .5

\title{
О ГЛАВНЫХ ФАКТОРАХ ПАРАБОЛИЧЕСКИХ МАКСИМАЛЬНЫХ ПОДГРУПП ГРУППЫ ${ }^{2} F_{4}\left(2^{2 n+1}\right)$
}

\author{
В. В. Кораблева
}

\begin{abstract}
Статья является продолжением работ автора, в которых было получено уточненное описание главных факторов параболической максимальной подгруппы, входящих в ее унипотентный радикал, для всех конечных простых групп лиева типа (нормальных и скрученных) за исключением групп ${ }^{2} F_{4}\left(2^{2 n+1}\right)$ и $B_{l}\left(2^{n}\right)$. В настоящей работе приводится такое описание для группы ${ }^{2} F_{4}\left(2^{2 n+1}\right)$. Доказана теорема, в которой для каждой параболической максимальной подгруппы группы ${ }^{2} F_{4}\left(2^{2 n+1}\right)$ дается фрагмент главного ряда, входящий в унипотентный радикал этой параболической подгруппы. Приводится таблица, в которой указываются порождающие элементы соответствующих главных факторов.
\end{abstract}

Ключевые слова: конечная простая группа, группа лиева типа, параболическая максимальная подгруппа, главный фактор, унипотентный радикал, усиленная версия гипотезы Симса.

V. V. Korableva. On chief factors of parabolic maximal subgroups of the group ${ }^{2} F_{4}\left(2^{2 n+1}\right)$.

This study continues the author's previous papers where a refined description of the chief factors of a parabolic maximal subgroup contained in its unipotent radical was obtained for all (normal and twisted) finite simple groups of Lie type except for the groups ${ }^{2} F_{4}\left(2^{2 n+1}\right)$ and $B_{l}\left(2^{n}\right)$. In present paper, such a description is given the group ${ }^{2} F_{4}\left(2^{2 n+1}\right)$. We prove a theorem in which, for every parabolic maximal subgroup of ${ }^{2} F_{4}\left(2^{2 n+1}\right)$, a fragment of the chief series contained in the unipotent radical of this subgroup is given. Generators of the corresponding chief factors are presented in a table.

Keywords: finite simple group, group of Lie type, parabolic maximal subgroup, chief factor, unipotent radical, strong version of the Sims conjecture.

MSC: 20D06, 20G41, 17B22

DOI: $10.21538 / 0134-4889-2019-25-4-99-106$

\section{Введение}

Одной из фундаментальных задач теории групп является изучение подгруппового строения групп. В постклассификационной теории конечных групп актуальными стали исследования подгрупп и представлений конечных простых групп. Группы лиева типа составляют основной массив конечных простых групп. Важный класс подстановочных представлений конечных групп лиева типа составляют их параболические представления, т.е. представления на смежных классах по параболическим подгруппам. В ранних работах автора было получено описание примитивных параболических подстановочных представлений всех групп лиева типа (нормальных и скрученных). В настоящее время автор продолжает исследование свойств таких представлений, а именно, изучает главные факторы параболических максимальных подгрупп конечных простых групп лиева типа. С использованием результатов из [9] автором в работах [4-8] было получено уточненное описание главных факторов параболической максимальной подгруппы, входящих в ее унипотентный радикал, для всех конечных простых групп лиева типа (нормальных и скрученных) за исключением групп ${ }^{2} F_{4}\left(2^{2 n+1}\right)$ и $B_{l}\left(2^{n}\right)$. В данной работе приводится такое описание для группы ${ }^{2} F_{4}\left(2^{2 n+1}\right)$. Заметим, что структура максимальных параболических подгрупп группы ${ }^{2} F_{4}\left(2^{2 n+1}\right)$ в терминах $(B, N)$-пар была описана в $[12, \S 10]$. Используя другой подход, автор доказывает следующую теорему в обозначениях разд. 1. 
Теорема. Пусть $G={ }^{2} F_{4}(q)$, где $q=2^{2 n+1} \geqslant 2, u P_{k}$ - параболическая максимальная подгруппа в $G$ для $k=1,2$. Тогда фрагмент главного рлда группы $P_{k}$, лежащий в ее унипотентном радикале $U_{k}$, при $k=1$ имеет вид $U_{1}=Y_{4}>Y_{3}>Y_{2}>Y_{1}>1$, где $\left|Y_{4} / Y_{3}\right|=\left|Y_{2} / Y_{1}\right|=q^{4}$, $\left|Y_{3} / Y_{2}\right|=\left|Y_{1}\right|=q$, a при $k=2$ имеет вид $U_{2}=Y_{5}>Y_{4}>Y_{3}>Y_{2}>Y_{1}>1$, где $\left|Y_{5} / Y_{4}\right|=$ $\left|Y_{3} / Y_{2}\right|=\left|Y_{1}\right|=q^{2},\left|Y_{4} / Y_{3}\right|=q^{4},\left|Y_{2} / Y_{1}\right|=q$. Кроме того, нижний центральный ряд группъ $U_{k}$ имеет вид $U_{1}>Y_{2}>Y_{1}>1$ при $k=1$ и $U_{2}>Y_{4}>Y_{3}>Y_{2}>Y_{1}>1$ nри $k=2$.

В конце статьи приводится таблица, в которой указываются порождающие элементы соответствующих главных факторов.

Результаты статьи будут использованы исследователями в доказательстве усиленной версии гипотезы Симса (см. [13]).

\section{1. Обозначения и вспомогательные результаты}

Используемые в статье обозначения из общей теории групп в основном стандартны, их можно найти в [11]. Напомним некоторые из них. Если $X$ и $Y-$ группы, а $n-$ натуральное число, то через $X . Y$ обозначается расширение группы $X$ при помощи группы $Y$, через $X^{n}-$ прямое произведение $n$ групп, каждая из которых изоморфна группе $X$, через $n-$ циклическая группа порядка $n$. Коммутатор элементов $x$ и $y$ группы обозначается через $[x, y]=x^{-1} y^{-1} x y$. Определения и обозначения, связанные с группами лиева типа, взяты из [10].

Пусть $G={ }^{2} F_{4}(q)$, где $q=2^{2 n+1} \geq 2$. Построение и свойства группы $G$ можно найти в $[15 ; 17]$ или [10]. Группа $G$ определяется как подгруппа группы Шевале $F_{4}(K)$, где $K=$ $G F(q)$, состоящая из всех элементов, неподвижных относительно некоторого автоморфизма группы $F_{4}(K)$.

Рассмотрим корневую систему $\Phi$ типа $F_{4}$ с фундаментальной системой корней $\pi=\left\{p_{1}, p_{2}\right.$, $\left.p_{3}, p_{4}\right\}$ и диаграммой Дынкина

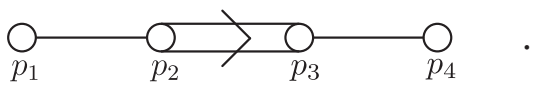

Тогда ввиду [1] множество $\Phi^{+}$положительных корней системы $\Phi$ относительно $\pi$ состоит из элементов

$$
\begin{array}{lll}
r_{1}=p_{1}, & r_{9}=p_{2}+2 p_{3}, & r_{17}=p_{1}+2 p_{2}+2 p_{3}+p_{4}, \\
r_{2}=p_{2}, & r_{10}=p_{2}+p_{3}+p_{4}, & r_{18}=p_{1}+p_{2}+2 p_{3}+2 p_{4}, \\
r_{3}=p_{3}, & r_{11}=p_{1}+p_{2}+2 p_{3}, & r_{19}=p_{1}+2 p_{2}+3 p_{3}+p_{4}, \\
r_{4}=p_{4}, & r_{12}=p_{1}+p_{2}+p_{3}+p_{4}, & r_{20}=p_{1}+2 p_{2}+2 p_{3}+2 p_{4}, \\
r_{5}=p_{1}+p_{2}, & r_{13}=p_{2}+2 p_{3}+p_{4}, & r_{21}=p_{1}+2 p_{2}+3 p_{3}+2 p_{4}, \\
r_{6}=p_{2}+p_{3}, & r_{14}=p_{1}+2 p_{2}+2 p_{3}, & r_{22}=p_{1}+2 p_{2}+4 p_{3}+2 p_{4}, \\
r_{7}=p_{3}+p_{4}, & r_{15}=p_{1}+p_{2}+2 p_{3}+p_{4}, & r_{23}=p_{1}+3 p_{2}+4 p_{3}+2 p_{4}, \\
r_{8}=p_{1}+p_{2}+p_{3}, & r_{16}=p_{2}+2 p_{3}+2 p_{4}, & r_{24}=2 p_{1}+3 p_{2}+4 p_{3}+2 p_{4} .
\end{array}
$$

Согласно [15] система $\Phi$ разбивается на шестнадцать классов:

$$
\begin{aligned}
& S_{1}=\left\{r_{8}, r_{16}, r_{21}, r_{24}\right\}, \quad S_{2}=\left\{r_{3}, r_{2}, r_{6}, r_{9}\right\}, \\
& S_{3}=\left\{r_{10}, r_{11}, r_{19}, r_{23}\right\}, \quad S_{4}=\left\{r_{7}, r_{5}, r_{12}, r_{18}\right\}, \\
& S_{5}=\left\{r_{17}, r_{22}\right\}, \quad S_{6}=\left\{r_{15}, r_{20}\right\}, \\
& S_{7}=\left\{r_{13}, r_{14}\right\}, \quad S_{8}=\left\{r_{4}, r_{1}\right\}, \\
& S_{-1}=\left\{-r_{8},-r_{16},-r_{21},-r_{24}\right\}, \quad S_{-2}=\left\{-r_{3},-r_{2},-r_{6},-r_{9}\right\}, \\
& S_{-3}=\left\{-r_{10},-r_{11},-r_{19},-r_{23}\right\}, \quad S_{-4}=\left\{-r_{7},-r_{5},-r_{12},-r_{18}\right\} \text {, } \\
& S_{-5}=\left\{-r_{17},-r_{22}\right\}, \quad S_{-6}=\left\{-r_{15},-r_{20}\right\}, \\
& S_{-7}=\left\{-r_{13},-r_{14}\right\}, \quad S_{-8}=\left\{-r_{4},-r_{1}\right\} \text {. }
\end{aligned}
$$


Пусть $\theta$ - автоморфизм поля $K$, возводящий его элементы в степень $2^{n}$. В [15] Ри ввел в рассмотрение следующие элементы $\alpha_{i}(t)$, где $i=1,2, \ldots, 12$ и $t \in K$, группы $G$ :

$$
\begin{array}{ll}
\alpha_{1}(t)=x_{r_{3}}\left(t^{\theta}\right) x_{r_{2}}(t) x_{r_{6}}\left(t^{\theta+1}\right), & \alpha_{2}(t)=x_{r_{6}}\left(t^{\theta}\right) x_{r_{9}}(t), \\
\alpha_{3}(t)=x_{r_{4}}\left(t^{\theta}\right) x_{r_{1}}(t), & \alpha_{4}(t)=x_{r_{7}}\left(t^{\theta}\right) x_{r_{5}}(t) x_{r_{12}}\left(t^{\theta+1}\right), \\
\alpha_{5}(t)=x_{r_{8}}\left(t^{\theta}\right) x_{r_{16}}(t) x_{r_{21}}\left(t^{\theta+1}\right), & \alpha_{6}(t)=x_{r_{10}}\left(t^{\theta}\right) x_{r_{11}}(t) x_{r_{19}}\left(t^{\theta+1}\right), \\
\alpha_{7}(t)=x_{r_{13}}\left(t^{\theta}\right) x_{r_{14}}(t), & \alpha_{8}(t)=x_{r_{12}}\left(t^{\theta}\right) x_{r_{18}}(t), \\
\alpha_{9}(t)=x_{r_{15}}\left(t^{\theta}\right) x_{r_{20}}(t), & \alpha_{10}(t)=x_{r_{17}}\left(t^{\theta}\right) x_{r_{22}}(t), \\
\alpha_{11}(t)=x_{r_{19}}\left(t^{\theta}\right) x_{r_{23}}(t), & \alpha_{12}(t)=x_{r_{21}}\left(t^{\theta}\right) x_{r_{24}}(t) .
\end{array}
$$

Каждому классу $S_{i}$ соответствует корневая подгруппа $X_{S_{i}}$ группы $G$. Корневые подгруппы $X_{S_{i}}$ для $1 \leq i \leq 8$ имеют вид

$$
\begin{array}{ll}
X_{S_{1}}=\left\langle\alpha_{5}(t), \alpha_{12}(u) \mid t, u, \in K\right\rangle, & X_{S_{5}}=\left\langle\alpha_{10}(t) \mid t \in K\right\rangle, \\
X_{S_{2}}=\left\langle\alpha_{1}(t), \alpha_{2}(u) \mid t, u \in K\right\rangle, & X_{S_{6}}=\left\langle\alpha_{9}(t) \mid t \in K\right\rangle, \\
X_{S_{3}}=\left\langle\alpha_{6}(t), \alpha_{11}(u) \mid t, u \in K\right\rangle, & X_{S_{7}}=\left\langle\alpha_{7}(t) \mid t \in K\right\rangle, \\
X_{S_{4}}=\left\langle\alpha_{4}(t), \alpha_{8}(u) \mid t, u \in K\right\rangle, & X_{S_{8}}=\left\langle\alpha_{3}(t) \mid t \in K\right\rangle .
\end{array}
$$

Корневые подгруппы $X_{S_{i}}$ для $1 \leq i \leq 8$ порождают максимальную унипотентную подгруппу $U$ группы $G$. Любой элемент $u$ из $U$ можно однозначно записать в виде $u=\prod_{i=1}^{12} \alpha_{i}\left(t_{i}\right)$, где $t_{i} \in K$. Подгруппа Бореля $B=N_{G}(U)$ группы $G$ является полупрямым произведением подгруппы $U$ и подгруппы Картана $H$ порядка $(q-1)^{2}$, изоморфной $K^{*} \times K^{*}$. Множество $\pi$ простых корней содержится в объединении классов $S_{2}$ и $S_{8}$, поэтому в группе $G$ имеется точно две параболические максимальные подгруппы, содержащие $B$, а именно $P_{1}=P_{S_{2}}=\left\langle B, X_{S_{-2}}\right\rangle$ и $P_{2}=P_{S_{8}}=\left\langle B, X_{S_{-8}}\right\rangle$. Чтобы определить строение подгруппы $U$, приведем следующий полный список коммутаторных соотношений между элементами вида $\alpha_{i}(t)$, где $1 \leq i \leq 12$ и $t \in K$ :

$$
\begin{aligned}
{\left[\alpha_{1}(t), \alpha_{3}(u)\right] } & =\alpha_{4}(t u) \alpha_{5}\left(t^{2 \theta+1} u^{2 \theta}\right) \alpha_{7}\left(t^{2 \theta+2} u\right) \alpha_{11}\left(t^{4 \theta+3} u^{2 \theta+1}\right) \alpha_{12}\left(t^{4 \theta+3} u^{2 \theta+2}\right), \\
{\left[\alpha_{1}(t), \alpha_{4}(u)\right] } & =\alpha_{5}\left(t u^{2 \theta}\right) \alpha_{6}\left(t^{2 \theta} u\right) \alpha_{7}\left(t^{2 \theta+1} u\right) \alpha_{9}\left(t u^{2 \theta+1}\right) \alpha_{10}\left(t^{2 \theta+1} u^{2 \theta+1}\right) \\
& \times \alpha_{11}\left(t^{2 \theta+2} u^{2 \theta+1}\right) \alpha_{12}\left(t^{2 \theta+1} u^{2 \theta+2}\right), \\
{\left[\alpha_{1}(t), \alpha_{6}(u)\right] } & =\alpha_{7}(t u), \quad\left[\alpha_{1}(t), \alpha_{10}(u)\right]=\alpha_{11}(t u), \\
{\left[\alpha_{1}(t), \alpha_{8}(u)\right] } & =\alpha_{9}(t u) \alpha_{11}\left(t^{2 \theta+2} u\right) \alpha_{12}\left(t^{2 \theta+1} u^{2 \theta}\right), \\
{\left[\alpha_{1}(t), \alpha_{9}(u)\right] } & =\alpha_{10}\left(t^{2 \theta} u\right) \alpha_{11}\left(t^{2 \theta+1} u\right) \alpha_{12}\left(t u^{2 \theta}\right), \\
{\left[\alpha_{2}(t), \alpha_{3}(u)\right] } & =\alpha_{5}\left(t u^{2 \theta}\right) \alpha_{6}(t u) \alpha_{7}\left(t^{2 \theta} u\right) \alpha_{8}\left(t u^{2 \theta+1}\right) \alpha_{9}\left(t^{2 \theta} u^{2 \theta+1}\right), \\
{\left[\alpha_{2}(t), \alpha_{4}(u)\right] } & =\alpha_{7}(t u) \alpha_{11}\left(t^{2 \theta} u^{2 \theta+1}\right) \alpha_{12}\left(t u^{2 \theta+2}\right), \\
{\left[\alpha_{2}(t), \alpha_{8}(u)\right] } & =\alpha_{10}(t u) \alpha_{11}\left(t^{2 \theta} u\right) \alpha_{12}\left(t u^{2 \theta}\right), \\
{\left[\alpha_{2}(t), \alpha_{9}(u)\right] } & =\alpha_{11}(t u), \quad\left[\alpha_{3}(t), \alpha_{5}(u)\right]=\alpha_{8}(t u), \\
{\left[\alpha_{3}(t), \alpha_{6}(u)\right] } & =\alpha_{8}\left(t^{2 \theta} u\right) \alpha_{9}\left(t u^{2 \theta}\right) \alpha_{12}\left(t u^{2 \theta+1}\right), \quad\left[\alpha_{3}(t), \alpha_{7}(u)\right]=\alpha_{9}\left(t^{2 \theta} u\right) \alpha_{10}\left(t u^{2 \theta}\right), \\
{\left[\alpha_{3}(t), \alpha_{11}(u)\right] } & =\alpha_{12}(t u), \quad\left[\alpha_{4}(t), \alpha_{5}(u)\right]=\alpha_{9}(t u), \\
{\left[\alpha_{4}(t), \alpha_{7}(u)\right] } & =\alpha_{10}\left(t^{2 \theta} u\right) \alpha_{11}\left(t u^{2 \theta}\right) \alpha_{12}\left(t^{2 \theta+1} u\right), \quad\left[\alpha_{4}(t), \alpha_{10}(u)\right]=\alpha_{12}(t u), \\
{\left[\alpha_{5}(t), \alpha_{6}(u)\right] } & =\alpha_{10}(t u), \quad\left[\alpha_{5}(t), \alpha_{7}(u)\right]=\alpha_{11}(t u), \\
{\left[\alpha_{6}(t), \alpha_{9}(u)\right] } & =\alpha_{12}(t u), \quad\left[\alpha_{7}(t), \alpha_{8}(u)\right]=\alpha_{12}(t u), \\
{\left[\alpha_{i}(t), \alpha_{i}(u)\right] } & =\alpha_{j(i)}\left(t^{2 \theta} u+t u^{2 \theta}\right),
\end{aligned}
$$

где $j(i)=2,8,12,11$ соответствуют $i=1,4,5,6$. Все другие коммутаторы $\left[\alpha_{i}(t), \alpha_{j}(u)\right]$ равны 1 . Кроме того, $\alpha_{i}(t) \alpha_{i}(u)=\alpha_{i}(t+u) \alpha_{j(i)}\left(t u^{2 \theta}\right)$ для $i=1,4,5,6$.

Отметим, что этот список приведен в работе [16], но с ошибкой в формуле для коммутатора $\left[\alpha_{2}(t), \alpha_{3}(u)\right]$ (см. [14, с. 57]). 


\section{2. Доказательство теоремы}

Опишем строение параболической максимальной подгруппы $P_{1}$, используя ее разложение Леви $P_{1}=U_{1} L_{1}$, где $U_{1}=\left\langle X_{S_{i}} \mid i \in\{1,3,4, \ldots, 8\}\right\rangle$ - унипотентный радикал и $L_{1}=$ $\left\langle H, X_{S_{2}}, X_{S_{-2}}\right\rangle$ - дополнение Леви в $P_{1}$. Рассматривая только те коммутаторные соотношения, в которых не встречаются множители $\alpha_{1}(t)$ и $\alpha_{2}(t)$, получаем, что $U_{1} \cong 2^{2 n+1} \cdot 2^{4(2 n+1)} \cdot 2^{5(2 n+1)}$. Подгруппа $L_{1}$ изоморфна прямому произведению группы ${ }^{2} B_{2}(q)$ и циклической группы порядка $q-1$ (см. [2]).

Пусть $Y_{1}=\left\langle\alpha_{12}(t) \mid t \in K\right\rangle$ и $Y_{2}=\left\langle\alpha_{i}\left(t_{i}\right) \mid t_{i} \in K, i \in\{8,9,10,11,12\}\right\rangle$. Тогда $Y_{1}$ является минимальной нормальной подгруппой, а $Y_{2}$ - нормальной подгруппой в $P_{1}$. Положим $\alpha_{-1}(t)=$ $x_{-r_{3}}\left(t^{\theta}\right) x_{-r_{2}}(t) x_{-r_{6}}\left(t^{\theta+1}\right)$ и $\alpha_{-2}(t)=x_{-r_{6}}\left(t^{\theta}\right) x_{-r_{9}}(t)$. Действие (сопряжением) группы $L_{1}$ на фактор-группу $Y_{2} / Y_{1}$ задается следующими коммутаторными соотношениями $(t, u \in K)$ :

$$
\begin{aligned}
& {\left[\alpha_{8}(t), \alpha_{1}(u)\right] Y_{1}=\alpha_{9}(t u) \alpha_{11}\left(t u^{2 \theta+2}\right) Y_{1},} \\
& {\left[\alpha_{9}(t), \alpha_{1}(u)\right] Y_{1}=\alpha_{10}\left(t u^{2 \theta}\right) \alpha_{11}\left(t u^{2 \theta+1}\right) Y_{1},} \\
& {\left[\alpha_{10}(t), \alpha_{1}(u)\right] Y_{1}=\alpha_{11}(t u) Y_{1},} \\
& {\left[\alpha_{8}(t), \alpha_{2}(u)\right] Y_{1}=\alpha_{10}(t u) \alpha_{11}\left(t u^{2 \theta}\right) Y_{1},} \\
& {\left[\alpha_{9}(t), \alpha_{2}(u)\right] Y_{1}=\alpha_{11}(t u) Y_{1},} \\
& {\left[\alpha_{9}(t), \alpha_{-1}(u)\right] Y_{1}=\alpha_{8}(t u) Y_{1},} \\
& {\left[\alpha_{10}(t), \alpha_{-1}(u)\right] Y_{1}=\alpha_{8}\left(t u^{2 \theta+1}\right) \alpha_{9}\left(t u^{2 \theta}\right) Y_{1},} \\
& {\left[\alpha_{11}(t), \alpha_{-1}(u)\right] Y_{1}=\alpha_{8}\left(t u^{2 \theta+2}\right) \alpha_{10}(t u) Y_{1},} \\
& {\left[\alpha_{10}(t), \alpha_{-2}(u)\right] Y_{1}=\alpha_{8}(t u) Y_{1},} \\
& {\left[\alpha_{11}(t), \alpha_{-2}(u)\right] Y_{1}=\alpha_{8}\left(t u^{2 \theta}\right) \alpha_{9}(t u) Y_{1},}
\end{aligned}
$$

поэтому $L_{1}$ действует неприводимо на $Y_{2} / Y_{1}$. Получаем фрагмент $Y_{2}>Y_{1}>1$ главного ряда группы $P_{1}$.

Действие (сопряжением) группы $L_{1}$ на фактор-группу $U_{1} / Y_{2}$ задается следующими соотношениями $(t, u \in K)$ :

$$
\begin{aligned}
& {\left[\alpha_{3}(t), \alpha_{1}(u)\right] Y_{2}=\alpha_{4}(t u) \alpha_{5}\left(t^{2 \theta} u^{2 \theta+1}\right) \alpha_{7}\left(t u^{2 \theta+2}\right) Y_{2},} \\
& {\left[\alpha_{4}(t), \alpha_{1}(u)\right] Y_{2}=\alpha_{5}\left(t^{2 \theta} u\right) \alpha_{6}\left(t u^{2 \theta}\right) \alpha_{7}\left(t u^{2 \theta+1}\right) Y_{2},} \\
& {\left[\alpha_{6}(t), \alpha_{1}(u)\right] Y_{2}=\alpha_{7}(t u) Y_{2},} \\
& {\left[\alpha_{3}(t), \alpha_{2}(u)\right] Y_{2}=\alpha_{5}\left(t^{2 \theta} u\right) \alpha_{6}(t u) \alpha_{7}\left(t u^{2 \theta}\right) Y_{2},} \\
& {\left[\alpha_{4}(t), \alpha_{2}(u)\right] Y_{2}=\alpha_{7}(t u) Y_{2},} \\
& {\left[\alpha_{4}(t), \alpha_{-1}(u)\right] Y_{2}=\alpha_{3}(t u) Y_{2},} \\
& {\left[\alpha_{6}(t), \alpha_{-1}(u)\right] Y_{2}=\alpha_{3}\left(t u^{2 \theta+1}\right) \alpha_{4}\left(t u^{2 \theta}\right) \alpha_{5}\left(t^{2 \theta} u\right) Y_{2},} \\
& {\left[\alpha_{7}(t), \alpha_{-1}(u)\right] Y_{2}=\alpha_{3}\left(t u^{2 \theta+2}\right) \alpha_{5}\left(t^{2 \theta} u^{2 \theta+1}\right) \alpha_{6}(t u) Y_{2},} \\
& {\left[\alpha_{6}(t), \alpha_{-2}(u)\right] Y_{2}=\alpha_{3}(t u) Y_{2},} \\
& {\left[\alpha_{7}(t), \alpha_{-2}(u)\right] Y_{2}=\alpha_{3}\left(t u^{2 \theta}\right) \alpha_{4}(t u) \alpha_{5}\left(t^{2 \theta} u\right) Y_{2} .}
\end{aligned}
$$

Это действие приводимо. Положим $Y_{3}=\left\langle\alpha_{i}\left(t_{i}\right) \mid t_{i} \in K, i \in\{5,8,9,10,11,12\}\right\rangle$. Тогда подгруппа $L_{1}$ нормализует $Y_{3}$ и действует неприводимо на факторах $U_{1} / Y_{3}$ и $Y_{3} / Y_{2}$. Получаем последний фрагмент $U_{1}=Y_{4}>Y_{3}>Y_{2}$ главного ряда группы $P_{1}$, лежащий в $U_{1}$.

Рассмотрим другую параболическую максимальную подгруппу $P_{2}$. Она имеет разложение Леви $P_{2}=U_{2} L_{2}$, где $\left.\left.U_{2}=\left\langle X_{S_{i}}\right| i \in\{1,2, \ldots, 7\}\right\}\right\rangle$ и $L_{2}=\left\langle H, X_{S_{8}}, X_{S_{-8}}\right\rangle$. Рассматривая только те коммутаторные соотношения, в которых не встречается множитель $\alpha_{3}(t)$, получаем, что $L_{2} \cong G L_{2}(q)$ и $U_{2} \cong 2^{2(2 n+1)} \cdot 2^{2 n+1} \cdot 2^{2(2 n+1)} \cdot 2^{4(2 n+1)} \cdot 2^{2(2 n+1)}$ (см. [3]). 
Из коммутаторных соотношений в группе $U_{2}$ следует, что подгруппа $Y_{1}=\left\langle\alpha_{i}\left(t_{i}\right)\right| t_{i} \in$ $K, i \in\{11,12\}\rangle$ является нормальной подгруппой в $P_{2}$. Положим $\alpha_{-3}(t)=x_{-r_{4}}\left(t^{\theta}\right) x_{-r_{1}}(t)$. Действие группы $L_{2}$ на $Y_{1}$ задается двумя соотношениями $(t, u \in K)$

$$
\left[\alpha_{11}(t), \alpha_{3}(u)\right]=\alpha_{12}(t u), \quad\left[\alpha_{12}(t), \alpha_{-3}(u)\right]=\alpha_{11}(t u) .
$$

Это действие неприводимо. Получаем фрагмент $Y_{1}>1$ главного ряда группы $P_{2}$.

Приведем нетривиальные коммутаторные соотношения в фактор-группе $U_{2} / Y_{1}(t, u \in K)$ :

$$
\begin{aligned}
{\left[\alpha_{1}(t), \alpha_{4}(u)\right] Y_{1} } & =\alpha_{5}\left(t u^{2 \theta}\right) \alpha_{6}\left(t^{2 \theta} u\right) \alpha_{7}\left(t^{2 \theta+1} u\right) \alpha_{9}\left(t u^{2 \theta+1}\right) \alpha_{10}\left(t^{2 \theta+1} u^{2 \theta+1}\right) Y_{1}, \\
{\left[\alpha_{1}(t), \alpha_{6}(u)\right] Y_{1} } & =\alpha_{7}(t u) Y_{1}, \quad\left[\alpha_{1}(t), \alpha_{8}(u)\right] Y_{1}=\alpha_{9}(t u) Y_{1}, \\
{\left[\alpha_{1}(t), \alpha_{9}(u)\right] Y_{1} } & =\alpha_{10}\left(t^{2 \theta} u\right) Y_{1}, \quad\left[\alpha_{2}(t), \alpha_{4}(u)\right] Y_{1}=\alpha_{7}(t u) Y_{1}, \\
{\left[\alpha_{2}(t), \alpha_{8}(u)\right] Y_{1} } & =\alpha_{10}(t u) Y_{1}, \quad\left[\alpha_{4}(t), \alpha_{5}(u)\right] Y_{1}=\alpha_{9}(t u) Y_{1}, \\
{\left[\alpha_{4}(t), \alpha_{7}(u)\right] Y_{1} } & =\alpha_{10}\left(t^{2 \theta} u\right) Y_{1}, \quad\left[\alpha_{5}(t), \alpha_{6}(u)\right] Y_{1}=\alpha_{10}(t u) Y_{1}, \\
{\left[\alpha_{1}(t), \alpha_{1}(u)\right] Y_{1} } & =\alpha_{2}\left(t^{2 \theta} u+t u^{2 \theta}\right) Y_{1}, \quad\left[\alpha_{4}(t), \alpha_{4}(u)\right] Y_{1}=\alpha_{8}\left(t^{2 \theta} u+t u^{2 \theta}\right) Y_{1} \text { и } \\
\alpha_{1}(t) \alpha_{1}(u) Y_{1} & =\alpha_{1}(t+u) \alpha_{2}\left(t u^{2 \theta}\right) Y_{1}, \quad \alpha_{4}(t) \alpha_{4}(u) Y_{1}=\alpha_{4}(t+u) \alpha_{8}\left(t u^{2 \theta}\right) Y_{1} .
\end{aligned}
$$

Рассмотрим подгруппу $Y_{2}=\left\langle\alpha_{i}\left(t_{i}\right) \mid t_{i} \in K, i \in\{10,11,12\}\right\rangle$. Фактор-группа $Y_{2} / Y_{1}$ является минимальной нормальной подгруппой в $P_{2} / Y_{1}$. Получаем фрагмент $Y_{2}>Y_{1}$ главного ряда группы $P_{2}$.

Приведем нетривиальные соотношения в фактор-группе $U_{2} / Y_{2}(t, u \in K)$ :

$$
\begin{aligned}
{\left[\alpha_{1}(t), \alpha_{4}(u)\right] Y_{2} } & =\alpha_{5}\left(t u^{2 \theta}\right) \alpha_{6}\left(t^{2 \theta} u\right) \alpha_{7}\left(t^{2 \theta+1} u\right) \alpha_{9}\left(t u^{2 \theta+1}\right) Y_{2}, \\
{\left[\alpha_{1}(t), \alpha_{6}(u)\right] Y_{2} } & =\alpha_{7}(t u) Y_{2}, \quad\left[\alpha_{1}(t), \alpha_{8}(u)\right] Y_{2}=\alpha_{9}(t u) Y_{2}, \\
{\left[\alpha_{2}(t), \alpha_{4}(u)\right] Y_{2} } & =\alpha_{7}(t u) Y_{2}, \quad\left[\alpha_{4}(t), \alpha_{5}(u)\right] Y_{2}=\alpha_{9}(t u) Y_{2}, \\
{\left[\alpha_{1}(t), \alpha_{1}(u)\right] Y_{2} } & =\alpha_{2}\left(t^{2 \theta} u+t u^{2 \theta}\right) Y_{2}, \quad\left[\alpha_{4}(t), \alpha_{4}(u)\right] Y_{2}=\alpha_{8}\left(t^{2 \theta} u+t u^{2 \theta}\right) Y_{2} \text { и } \\
\alpha_{1}(t) \alpha_{1}(u) Y_{2} & =\alpha_{1}(t+u) \alpha_{2}\left(t u^{2 \theta}\right) Y_{2}, \quad \alpha_{4}(t) \alpha_{4}(u) Y_{2}=\alpha_{4}(t+u) \alpha_{8}\left(t u^{2 \theta}\right) Y_{2} .
\end{aligned}
$$

Рассмотрим нормальную в $P_{2}$ подгруппу $Y_{3}=\left\langle\alpha_{i}\left(t_{i}\right) \mid t_{i} \in K, i \in\{7,9,10,11,12\}\right\rangle$. Действие группы $L_{2}$ на $Y_{3} / Y_{2}$ задается соотношениями $(t, u \in K)$

$$
\left[\alpha_{7}(t), \alpha_{3}(u)\right] Y_{2}=\alpha_{9}\left(t u^{2 \theta}\right) Y_{2}, \quad\left[\alpha_{9}(t), \alpha_{-3}(u)\right] Y_{2}=\alpha_{7}(t u) Y_{2} .
$$

Это действие неприводимо. Получаем фрагмент $Y_{3}>Y_{2}$ главного ряда группы $P_{2}$.

Приведем нетривиальные коммутаторные соотношения в фактор-группе $U_{2} / Y_{3}(t, u \in K)$ :

$$
\begin{aligned}
{\left[\alpha_{1}(t), \alpha_{4}(u)\right] Y_{3} } & =\alpha_{5}\left(t u^{2 \theta}\right) \alpha_{6}\left(t^{2 \theta} u\right) Y_{3}, \quad\left[\alpha_{1}(t), \alpha_{1}(u)\right] Y_{3}=\alpha_{2}\left(t^{2 \theta} u+t u^{2 \theta}\right) Y_{3}, \\
{\left[\alpha_{4}(t), \alpha_{4}(u)\right] Y_{3} } & =\alpha_{8}\left(t^{2 \theta} u+t u^{2 \theta}\right) Y_{3} \text { и } \\
\alpha_{1}(t) \alpha_{1}(u) Y_{3} & =\alpha_{1}(t+u) \alpha_{2}\left(t u^{2 \theta}\right) Y_{3}, \quad \alpha_{4}(t) \alpha_{4}(u) Y_{3}=\alpha_{4}(t+u) \alpha_{8}\left(t u^{2 \theta}\right) Y_{3} .
\end{aligned}
$$

Рассмотрим подгруппу $Y_{4}=\left\langle\alpha_{i}\left(t_{i}\right) \mid t_{i} \in K, i \in\{2,5,6,7,8,9,10,11,12\}\right\rangle$, нормальную в $P_{2}$. Неприводимое действие $L_{2}$ на фактор-группу $Y_{4} / Y_{3}$ задается соотношениями $(t, u \in K)$

$$
\begin{aligned}
{\left[\alpha_{2}(t), \alpha_{3}(u)\right] Y_{3} } & =\alpha_{5}\left(t u^{2 \theta}\right) \alpha_{6}(t u) \alpha_{8}\left(t u^{2 \theta+1}\right) Y_{3}, \quad\left[\alpha_{6}(t), \alpha_{3}(u)\right] Y_{3}=\alpha_{8}\left(t u^{2 \theta}\right) Y_{3}, \\
{\left[\alpha_{5}(t), \alpha_{3}(u)\right] Y_{3} } & =\alpha_{8}(t u) Y_{3}, \quad\left[\alpha_{5}(t), \alpha_{-3}(u)\right] Y_{3}=\alpha_{2}\left(t u^{2 \theta}\right) Y_{3}, \\
{\left[\alpha_{6}(t), \alpha_{-3}(u)\right] Y_{3} } & =\alpha_{2}(t u) Y_{3}, \quad\left[\alpha_{8}(t), \alpha_{-3}(u)\right] Y_{3}=\alpha_{2}\left(t u^{2 \theta+1}\right) \alpha_{5}(t u) \alpha_{6}\left(t u^{2 \theta}\right) Y_{3} .
\end{aligned}
$$

Получаем фрагмент $Y_{4}>Y_{3}$ главного ряда группы $P_{2}$. 
Главные факторы параболических максимальных подгрупп в ${ }^{2} F_{4}\left(2^{2 n+1}\right)$

\begin{tabular}{|l|l|l|}
\hline$P_{k}$ & $Y_{j+1} / Y_{j}$ & $\alpha_{i}\left(t_{i}\right): Y_{j+1} / Y_{j}=\prod_{i}\left\langle\alpha_{i}\left(t_{i}\right) \mid t_{i} \in K\right\rangle Y_{j} / Y_{j}$ \\
\hline$P_{1}$ & $Y_{4} / Y_{3}$ & $\alpha_{3}\left(t_{3}\right), \alpha_{4}\left(t_{4}\right), \alpha_{6}\left(t_{6}\right), \alpha_{7}\left(t_{7}\right)$ \\
\hline$P_{1}$ & $Y_{3} / Y_{2}$ & $\alpha_{5}\left(t_{5}\right)$ \\
\hline$P_{1}$ & $Y_{2} / Y_{1}$ & $\alpha_{8}\left(t_{8}\right), \alpha_{9}\left(t_{9}\right), \alpha_{10}\left(t_{10}\right), \alpha_{11}\left(t_{11}\right)$ \\
\hline$P_{1}$ & $Y_{1} / 1$ & $\alpha_{12}\left(t_{12}\right)$ \\
\hline$P_{2}$ & $Y_{5} / Y_{4}$ & $\alpha_{1}\left(t_{1}\right), \alpha_{4}\left(t_{4}\right)$ \\
\hline$P_{2}$ & $Y_{4} / Y_{3}$ & $\alpha_{2}\left(t_{2}\right), \alpha_{5}\left(t_{5}\right), \alpha_{6}\left(t_{6}\right), \alpha_{8}\left(t_{8}\right)$ \\
\hline$P_{2}$ & $Y_{3} / Y_{2}$ & $\alpha_{7}\left(t_{7}\right), \alpha_{9}\left(t_{9}\right)$ \\
\hline$P_{2}$ & $Y_{2} / Y_{1}$ & $\alpha_{10}\left(t_{10}\right)$ \\
\hline$P_{2}$ & $Y_{1} / 1$ & $\alpha_{11}\left(t_{11}\right), \alpha_{12}\left(t_{12}\right)$ \\
\hline
\end{tabular}

Подгруппа $U_{2} / Y_{4}$ нормальна в $P_{2} / Y_{4}$. Неприводимое действие группы $L_{2}$ на эту подгруппу задается двумя коммутаторными соотношениями $(t, u \in K)$

$$
\left[\alpha_{1}(t), \alpha_{3}(u)\right] Y_{4}=\alpha_{4}(t u) Y_{4}, \quad\left[\alpha_{4}(t), \alpha_{-3}(u)\right] Y_{4}=\alpha_{1}(t u) Y_{4},
$$

a $U_{2}=Y_{5}>Y_{4}$ - последний фрагмент главного ряда группы $P_{2}$, содержащийся в унипотентном радикале $U_{2}$.

Утверждение теоремы о нижнем центральном ряде группы $U_{k}$ легко следует из приведенных выше рассуждений.

Теорема доказана.

Занесем полученные при доказательстве теоремы результаты в таблицу (см. выше). Во втором столбце таблицы укажем главные факторы $Y_{j+1} / Y_{j}$ фрагмента главного ряда, входящего в унипотентный радикал, каждой параболической максимальной подгруппы $P_{k}$, указанной в первом столбце. Третий столбец нашей таблицы содержит порождающие элементы $\alpha_{i}\left(t_{i}\right) Y_{j}$ главного фактора $Y_{j+1} / Y_{j}=\prod_{i} U_{i} Y_{j} / Y_{j}$, где $U_{i}=\left\langle\alpha_{i}(t) \mid t \in K\right\rangle$.

\section{СПИСОК ЛИТЕРАТУРЫ}

1. Бурбаки Н. Группы и алгебры Ли. Гл. VII-VIII. М.: Мир, 1978. 342 с.

2. Васильев А.В. Минимальные подстановочные представления конечных простых исключительных групп скрученного типа // Алгебра и логика. 1998 Т. 37, № 1. С. 17-35.

3. Кораблева В.В. Параболические подстановочные представления групп ${ }^{2} F_{4}(q)$ and ${ }^{3} D_{4}\left(q^{3}\right) / /$ Мат. заметки. 2000. Т. 67, № 1. С. 69-76. doi 10.4213/mzm815.

4. Кораблева В.В. О главных факторах параболических максимальных подгрупп конечных простых групп нормального лиева типа // Сиб. мат. журн. 2014. Т. 55, № 4. С. 764-782.

5. Кораблева В.В. О главных факторах параболических максимальных подгрупп группы ${ }^{2} E_{6}\left(q^{2}\right) / /$ Алгебра и математическая логика, теория и приложения: тез. Междунар. конф. Казань, 2014. C. $82-83$.

6. Кораблева В.В. О главных факторах параболических максимальных подгрупп группы ${ }^{3} D_{4}\left(q^{3}\right)$ // Мальцевские чтения: тез. Междунар. конф., посвящ. 75-летию Ю. Л. Ершова. Новосибирск, 2015. С. 106.

7. Кораблева В.В. О главных факторах параболических максимальных подгрупп скрученных классических групп // Сиб. мат. журн. 2015. Т. 56, № 5. С. 1100-1110. doi 10.17377/smzh.2015.56.510 .

8. Кораблева В.В. О главных факторах параболических максимальных подгрупп специальных конечных простых групп исключительного лиева типа // Сиб. мат. журн. 2017. Т. 58, № 6. C. 1332-1340. doi 10.17377/smzh.2017.58.612 .

9. Azad H., Barry M., Seitz G. On the structure of parabolic subgroup // Com. in Algebra. 1990. Vol. 18, no. 2. P. 551-562. doi 10.1080/00927879008823931.

10. Carter R.W. Simple groups of Lie type. London: John Wiley and Sons, 1972. 332 p. ISBN: 0471137359.

11. Conway J.H., Curtis R.T., Norton S.P., Parker R.A., Wilson R.A. Atlas of finite groups. Oxford: Clarendon Press, 1985. 252 p. ISBN: 0198531990. 
12. Fong P., Seitz G. Groups with (B, N)-pair of rank 2, II // Invent. Math. 1974. Vol. 24. P. 191-239.

13. Kondrat'ev A.S., Trofimov V.I. Vertex stabilizers of vertices of graphs with primitive automorphism groups and a strong version of the Sims conjecture // Proc. Conf. "Groups St Andrews 2017 in Birmingham". Cambridge: Cambridge Univ. Press, 2019. P. 419-426. (London Math. Soc. Note Ser. 2019; vol. 455.)

14. Malle G. The maximal subgroups of ${ }^{2} F_{4}\left(q^{2}\right) / /$ J. Algebra. 1991. Vol. 139. P. 52-69. doi 10.1016/0021-8693(91)90283-E .

15. Ree R. A family of simple groups associated with simple Lie algebra type $F_{4} / /$ Am. J. Math. 1961. Vol. 83. P. 401-420. doi 10.2307/2372886.

16. Shinoda K. A characterization of odd order extensions of the Ree groups ${ }^{2} F_{4}(q) / /$ J. Fac. Sci. Univ. 1975. Vol. 22. P. 79-102.

17. Tits J. Algebraic and abstract simple groups // Ann. of Math. 1964. Vol. 80. P. 313-329. doi $10.2307 / 1970394$.

Поступила 7.11.2019

После доработки 22.11.2019

Принята к публикации 25.11.2019

Кораблева Вера Владимировна

д-р физ.-мат. наук, доцент

профессор кафедры КБ и ПА

Челябинский государственный университет

г. Челябинск;

ведущий науч. сотрудник

Институт математики и механики им. Н. Н. Красовского УрО РАН

г. Екатеринбург

e-mail: vvki@csu.ru

\section{REFERENCES}

1. Bourbaki N. Groupes et algèbres de Lie (Chapt. IV-VI). Paris: Hermann, 1968, 282 p. doi: 10.1007/9783-540-34491-9. Translated to Russian under the title Gruppy i algebry Li (glavy IV - VI). Moscow: Mir Publ., 1972, 334 p.

2. Vasil'ev A.V. Minimal permutation representations of finite simple exceptional twisted groups. Algebra and Logic, 1998, vol. 37, no. 1, pp. 9-20. doi 10.1007/BF02684081.

3. Korableva V.V. Parabolic permutation representations of the groups ${ }^{2} F_{4}(q)$ and ${ }^{3} D_{4}\left(q^{3}\right)$. Math. Notes, 2000, vol. 67, no. 1, pp. 55-60. doi: 10.1007/BF02675792.

4. Korableva V.V. On the chief factors of parabolic maximal subgroups of finite simple groups of normal Lie type. Sib. Math. J., 2014, vol. 55, no. 4, pp. 622-638. doi: 10.1134/S0037446614040053.

5. Korableva V.V. On chief factors of parabolic maximal subgroups of the group ${ }^{2} E_{6}\left(q^{2}\right)$. International Conf. Algebra and Mathematical Logic: Theory and Applications, Collection of Abstracts, Kazan: 2014, pp. 82-83 (in Russian).

6. Korableva V.V. On chief factors of parabolic maximal subgroups of the group ${ }^{3} D_{4}\left(q^{3}\right)$. International Conf. Mal'tsev Meeting dedicated to 75th anniversary of Yuri L. Ershov, Collection of Abstracts, Novosibirsk: 2015. P. 106 (in Russian).

7. Korableva V.V. On the chief factors of maximal parabolic subgroups of twisted classical groups. Sib. Math. J., 2015, vol. 56, no. 5, pp. 879-887. doi 10.1134/S0037446615050109.

8. Korableva V.V. On the chief factors of parabolic maximal subgroups of special finite simple groups of exceptional Lie type. Sib. Math. J., 2017, vol. 58, no. 6, pp. 1034-1041. doi 10.1134/S003744661706012X.

9. Azad H., Barry M., Seitz G. On the structure of parabolic subgroup. Comm. Algebra. 1990. vol. 18, no. 2. pp. 551-562. doi 10.1080/00927879008823931.

10. Carter R.W. Simple groups of Lie type. London: John Wiley and Sons, 1972. 331 p.

11. Conway J.H., Curtis R.T., Norton S.P., Parker R.A., Wilson R.A. Atlas of finite groups. Oxford: Clarendon Press, 1985, 252 p. ISBN: 0198531990.

12. Fong P., Seitz G. Groups with (B,N)-pair of rank 2, II . Invent.Math. 1974. vol. 24. pp. 191-239. 
13. Kondrat'ev A.S., Trofimov V.I. Vertex stabilizers of vertices of graphs with primitive automorphism groups and a strong version of the Sims conjecture. Proc. conf. "Groups St Andrews 2017 in Birmingham". Cambridge: Cambridge University Press, 2019 (London Math. Soc. Note Ser. 2019. Vol. 455. pp. 419-426.)

14. Malle G. The Maximal subgroups of ${ }^{2} F_{4}\left(q^{2}\right)$ J. Algebra, 1991, vol. 139, pp. 52-69. doi 10.1016/0021-8693(91)90283-E .

15. Ree R. A family of simple groups associated with simple Lie algebra type $F_{4}$. Amer. J. Math., 1961, vol. 83, pp. 401-420. doi 10.2307/2372886.

16. Shinoda K. A characterization of odd order extensions of the Ree groups ${ }^{2} F_{4}(q)$. J. Fac. Sci. Univ, 1975, vol. 22, pp. 79-102.

17. Tits J. Algebraic and abstract simple groups. Ann. of Math., 1964, vol. 80, pp. 313-329. doi 10.2307/1970394.

Received November 11, 2019

Revised November 22, 2019

Accepted November 25, 2019

Vera Vladimirovna Korableva, Dr. Phys.-Math. Sci., Chelyabinsk State University, Chelyabinsk, 45400 Russia; Krasovskii Institute of Mathematics and Mechanics of the Ural Branch of the Russian Academy of Sciences, Yekaterinburg, 620108 Russia, e-mail: vvk@csu.ru .

Cite this article as: V. V. Korableva. On the chief factors of parabolic maximal subgroups of the group ${ }^{2} F_{4}\left(2^{2 n+1}\right)$, Trudy Instituta Matematiki i Mekhaniki URO RAN, 2019, vol. 25, no. 4, pp. 99-106 . 